\title{
A biomechanical study of the role of sitagliptin on the bone characteristics of diabetic rats
}

\author{
Stavros K. Kourkoulis, Ermioni D. Pasiou \\ National Technical University of Athens, Laboratory for Testing and Materials, Unit of Biomechanics, Greece \\ stakkoun@central.ntua.gr, bttps:/ / orcid.org/0000-0003-3246-9308 \\ epasion@teemail.gr, https://orcid.org/0000-0003-1580-3415
}

Arezoo Abdi, Despoina N. Perrea

National and Kapodistrian University of Athens, Medical School, Laboratory for Experimental Surgery \& Surgical Research "N.S. Christeas", Greece

aabdi@med.uoa.gr,dperrea@med.uoa.gr

John Vlamis

University of Athens, KAT General Hospital, $3^{\text {rd }}$ Orthopaedic Department, Greece

jvlamis@email.com

\begin{abstract}
An experimental protocol is described aiming to explore the influence of Type 2 Diabetes Mellitus on the biomechanical response of the bone tissue and, also, to quantify the potential beneficial role of a pharmaceutical treatment, based on sitagliptin, a diabetes drug that increases the levels of natural substances called incretins. Twenty eight male, 10-week old Wistar rats were used, divided into three groups, i.e., the control one, the group including the diabetic rats and, finally, the group including the diabetic rats which were treated using sitagliptin. The biomechanical study was based on a series of three-point bending tests of the femora of the sacrificed rats and the analysis of the experimental data was implemented in terms of the actual geometry of the fractured cross-section. It was concluded that diabetic bones undertake larger forces despite the fact that the "diameter" of their crosssection was somehow smaller. On the contrary, the slope of the load-deflection curve (corresponding to a measure of the stiffness) of diabetic bones is slightly lower compared to the control bones. Finally, it seems that treating diabetic animals with sitagliptin only partly reverses the effect of Type 2 Diabetes Mellitus on their bone tissue, at least concerning its strength and stiffness.
\end{abstract}

KEYwORDs. Male Wistar rats; Type-2 diabetes; Sitagliptin; Biomechanics; Three point bending; Video-extensometer.

\section{OPEN ACCESS}

Citation: Kourkoulis, S.K., Pasiou, E.D., Abdi, A., Perrea, D.N., Vlamis, J., A biomechanical study of the role of sitagliptin on the bone characteristics of diabetic rats, Frattura ed Integrità Strutturale, 51 (2020) 127-135.

Received: 13.10 .2019

Accepted: 05.11.2019

Published: 01.01.2020

Copyright: (c) 2020 This is an open access article under the terms of the CC-BY 4.0, which permits unrestricted use, distribution, and reproduction in any medium, provided the original author and source are credited. 


\section{INTRODUCTION}

$\mathrm{D}$ iabetes mellitus is a disease which increases the blood glucose levels of the patient. There are mainly two types of diabetes, i.e., Type 1 (insulin is not produced at all) and Type 2 (the total amount of insulin required cannot be produced). Insulin is a hormone produced in the pancreas and its role is to move glucose from the blood into cells, regulating blood sugar levels. The amount of insulin released is augmented by metabolic hormones, the incretins, which are some of the gut hormones (secreted after glucose consumption) stimulating insulin secretion together with hyperglycemia. Glucose-dependent Insulinotropic Polypeptide (GIP) and Glucagon-Like Peptide-1 (GLP-1) are known incretin hormones from the upper (GIP, K cells) and lower (GLP-1, L cells) gut [1]. These two hormones together create the incretin effect, namely, a two- to three-fold higher insulin secretory response to oral as compared to intravenous glucose administration. It is known that, in subjects with Type 2 diabetes this incretin effect is diminished or even no longer present. Once released to the blood stream, these two hormones are rapidly degraded by an endopeptidase, the dipeptidylpeptidase 4 (DPP-4) [1]. Glucagon-like peptide-1 receptor agonists (GLP-1 RA) and dipeptidyl peptidase-4 inhibitors (DPP-4i) are new incretin-based drugs which have emerged as a potentially new class of drugs for the management of the Type 2 Diabetes Mellitus (T2DM), and were received with enthusiasm by the medical community due to their extra-pancreatic effects.

The present study focuses on T2DM, taking into account that T2DM patients are the majority $(>90 \%)$ of diabetic patients, where inadequate insulin is produced, less sugar is moved into cells and therefore blood glucose levels increase. Nowadays, plenty of drugs are used for the treatment of diabetic patients, including DPP-4 inhibitors, such as sitagliptin used in this protocol, which blocks the enzyme dipeptidyl peptidase-4 (DPP-4), delaying the deactivation of incretins.

During the last decades there has been increased interest in the relationship of bone metabolism with diabetes. Due to the increased prevalence of diabetes mellitus globally, research on some of the lesser-known effects, including impaired bone health, is gaining a lot of attention. The actual effect of diabetes on bone fragility and fractures, as well as the implications of various therapeutic strategies on the bone, remain unclear [2, 3]. All studies agree that patients with T2DM have 40-70\% increased fracture risk, however their bone mineral density is normal or increased. This suggests that other factors, besides bone quality, affect bone fragility $[4,5]$. Animal and human diabetic subjects have consistently lower bone formation, however the findings on bone resorption are not so clear. Most animal studies reveal increased bone resorption in diabetic subjects, whereas clinical studies show decreased bone turnover in general $[6,7]$.

In the direction of gaining deeper insight in this issue, an experimental protocol is presented in this study, aiming to study the influence of T2DM on the biomechanical response of the bone tissue and, also, to quantify the potential beneficial role of a pharmaceutical treatment, based on sitagliptin. As with most similar studies, the fracture load is considered as the criterion for assessing the quality of bone tissue. It is mentioned, however, that the load, expressed in terms of the fracture force, does not take into consideration the geometrical characteristics and anatomic variations of bones, quite often shadowing the conclusions of biomechanical studies. In fact, from the Mechanics of Materials point of view, it is the fracture stress rather than the fracture force that characterizes the strength of any given material.

In this context, the fracture stress, as obtained from series of three-point bending tests, is used for the quantification of the role of T2DM and sitagliptin on the mechanical quality of bone tissue. The specific test is chosen due to its simplicity and, also, due to its wide application in similar protocols worldwide. The main challenge of the study was the accurate determination of the geometrical features of the fractured cross-section, in an attempt to avoid adopting simplifying assumptions, like, for example, to consider the cross-section as a circular or elliptic ring. In this direction, the approach suggested by Biewener [8] (permitting determination of the area, the centroid, the second moment of area and the coordinates of the points with the maximum distance from the neutral plane), was adopted and developed further, for the calculation of the fracture stress. The study enlightens interesting aspects of the role of T2DM and sitagliptin on the quality of the bone tissue of diabetes patients, which are not always in line with the respective ones of already published studies.

\section{THE EXPERIMENTAL PROTOCOL}

\section{Animals}

7 wenty eight (28) male 10-week old Wistar rats were used in the present study. The animals were divided into three (3) groups: (a) the control (C) one, (b) the diabetic (D) one and (c) the diabetic one, the animals of which after having become diabetic were treated using sitagliptin (D-S). More specifically, twenty (20) of the rats were made diabetic by adding fructose in their drinking water and, also, by injecting streptozotocin (STZ) [9]. On the contrary, the rats of the control group were drinking normal tap water and they received a vehicle buffer injection (in other words, they received a normal saline injection). 
One week after the injection, the animals with non-fasting blood glucose levels higher than $300 \mathrm{mg} / \mathrm{dl}$ were considered diabetic. The injection was repeated if necessary. As a next step and after diabetes was confirmed, twelve (12) of the diabetic rats were treated with sitagliptin per os for eight (8) weeks. The remaining eight (8) diabetic rats and the control ones did not receive any drug treatment. Tab. 1 recapitulates the three groups of animals, as well as the treatment offered to each group. All animals were fed normal pellet diet and eight (8) weeks after their 8-week treatment they were sacrificed.

It should be underlined that during the whole protocol, all regulations for animal protocols were followed (i.e., all animal procedures were approved by the National Bioethics Council of the Greek Ministry of Education).

\begin{tabular}{|c|c|c|c|c|}
\hline Group & Number of rats & Water & Injection & Treatment \\
\hline Control (C) & 8 & Normal drinking water & Vehicle buffer injection (ip) & - \\
\hline Diabetic-Sitagliptin (D-S) & 12 & $\begin{array}{l}\text { Water with } 10 \% \\
\text { frustose ad libitum }\end{array}$ & $\begin{array}{l}\text { Single injection (ip) of } \\
\text { streptozotocin (STZ) } \\
\text { ( } 40 \mathrm{mg} / \mathrm{kg} \text { b.w.) dissolved } \\
\text { in citrate buffer (pH } 4.4 \text { ) }\end{array}$ & $\begin{array}{l}\text { Sitagliptin per os } \\
(10 \mathrm{mg} / \mathrm{kg} / \text { day })\end{array}$ \\
\hline
\end{tabular}

Table 1: The groups of animals tested and the treatment taken.

\section{Experimental procedure}

The sacrifices of the animals were made in the "Laboratory for Experimental Surgery \& Surgical Research N. S. Christeas" of the "National and Kapodistrian University of Athens". After each sacrifice, the right femur of the rat was removed and it was transferred in normal saline solution to the "Laboratory for Testing and Materials" of the "National Technical University of Athens" within the next 30 minutes, in order to study their mechanical behaviour under three point bending.

All experiments were carried out using an MTS electromechanical loading frame and a specially designed apparatus for supporting the bones (Figs.1(a,b)) dictated by the relatively small size of the femora. The distance between the supports was set equal to $20 \mathrm{~mm}$ and it was kept constant in all experiments. The load was applied monotonically up to the fracture of the specimens with the aid of a punch of rounded tip. All tests were carried out under displacement-control mode, at a constant rate of $0.5 \mathrm{~mm} / \mathrm{min}$, simulating quasi-static loading conditions. The deflection of the bones at their mid-span was measured with the aid of a contactless video-extensometer by Limess (Fig.1c). The advantage of using the specific technique will be discussed in next section.

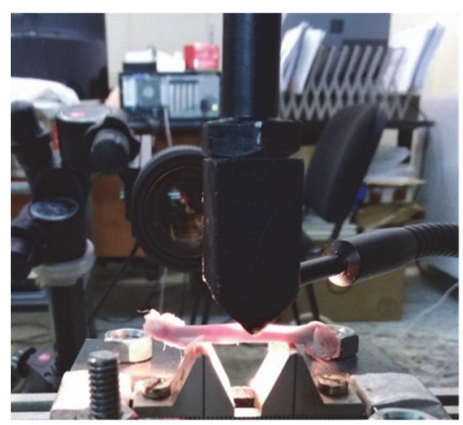

(a)

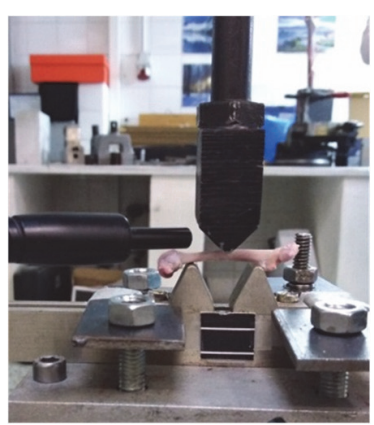

(b)

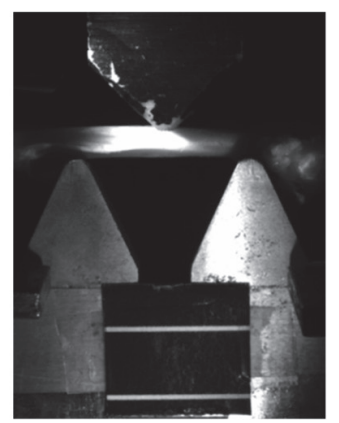

(c)

Figure 1: (a,b) Both sides of the experimental set-up used; (c) A typical image taken by the video-extensometer during the experiment.

The perimeter of the cross-section of the diaphysis of long bones is usually of irregular shape, as it can be clearly seen in Figs.2(a-c). It is only in very few cases that the perimeter can be considered (with a satisfactory level of approximation) of elliptical shape (see the white dotted ellipse in Fig.2d). Therefore, the exact settlement of the bone on the supports is not a priori known. This means that, for the majority of specimens, the loading axis does neither coincide with one of the two principal axes of the cross-section nor it passes through the centroid of the cross-section (generating, thus, shear stresses, in addition to the normal ones). Furthermore, and taking into account that all geometrical characteristics of the cross-sectional area (like for example the tensor of the second moments of area) are unknown, calculating the magnitude of the stresses developed (normal ones due to bending and shear ones due to parasitic torsion) becomes a challenging task.

To cope with these problems, the procedure proposed by Biewener's [8], as it was refined by Kourkoulis et al. [10], was adopted. As a first step, the bone was placed on the supports of the experimental apparatus and a pre-load (lower than $5 \mathrm{~N}$, i.e., lower than $3 \%$ of the maximum load undertaken by the specimens) was applied on the femur in order to stabilize it. In 
this position, the point of the bone at which the tip of the loading punch was coming in contact, was marked red. Then, the respective point at the lower level of the femur was marked black, as it can be seen in Fig.2e for the cross-section at the midspan of a typical femur. The specific procedure permitted determination of the direction of the loading axis.

After the end of the experiments, one of the two fractured parts of each specimen was placed vertically in a plastic cup which was then filled with molten resin (Fig.3a). After the curing time of the resin, as it is dictated by the manufacturer, the construct, i.e., the bone and the surrounding resin, was removed from the cup (Figs.3(b,c)). The free surface of the construct was then grinded by means of a series of abrasive papers of increasing smoothness up to the level of the marked points mentioned in the previous paragraph (i.e., the marks determining the loading axis). Finally, the polished surface of the construct were photographed with the aid of a stereoscope (Figs.2(a-d) and Fig.3d).

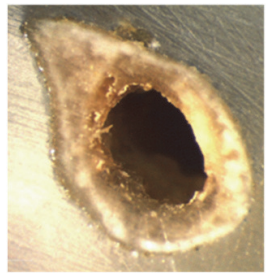

(a)

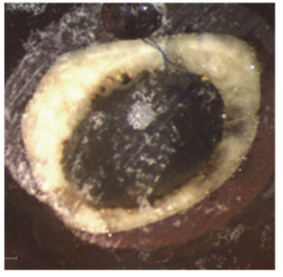

(b)

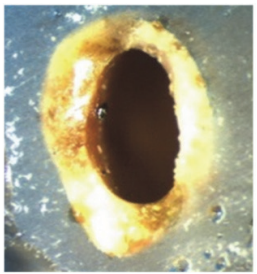

(c)

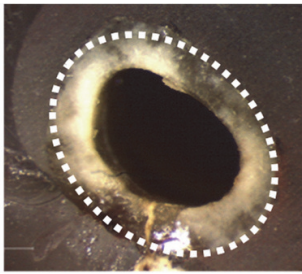

(d)

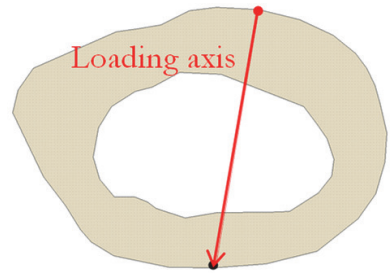

(e)

Figure 2: (a-c) Typical cross-sections of the bones tested; (d) The approximation of the cross-section using an ellipse; (e) The loading axis as it was determined for a typical cross-section of a femur.

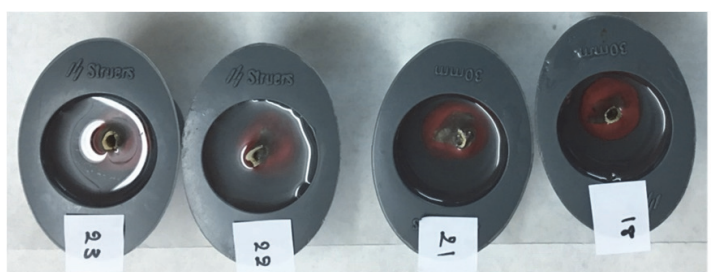

(a)

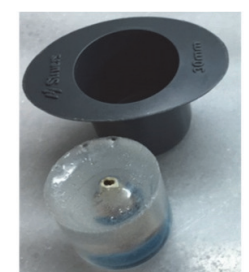

(b)

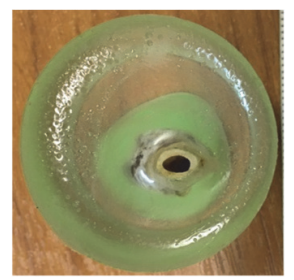

(c)

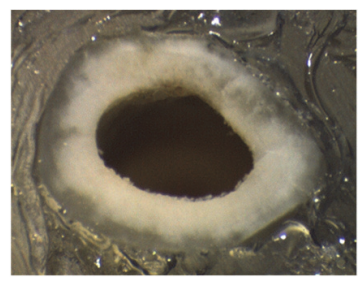

(d)

Figure 3: (a) The fractured bones surrounded by the resin in the plastic caps; (b,c) Typical bone-resin construct; (d) Photo of the polished surface of a typical construct.

\section{Obtaining the geometrical data of the fractured cross-sections of the specimens}

Each photo taken by the stereoscope was imported in the AutoCAD software and both the outer and inner perimeters of the femur's fractured cross-section were drawn, together with the line corresponding to the loading axis, as it was indicated by the previously mentioned coloured marks (Fig.4a). Taking advantage of these drawings, the cross-sectional area, the centroid of the cross-section (and therefore the eccentricity of the loading axis with respect to the centroid, e), the average thickness of the cortical bone and the area enclosed by the median line were calculated (Figs.4(b,c)) for each specimen. Having determined the centroid of the cross-section, a system $\mathrm{x}_{\mathrm{c}} \mathrm{C}_{\mathrm{c}}$ is initially chosen, with the $\mathrm{y}_{\mathrm{c}}$-axis to be parallel to the loading axis (Fig.5a) and the second moment of area about the centroidal axes were calculated, i.e., $\mathrm{I}_{\mathrm{xcxc}}, \mathrm{I}_{\mathrm{ycyc}}, \mathrm{I}_{\mathrm{xcyc}}$. As a next step, the principal second moments of area were determined, i.e., $I_{x p x p}=I_{\min }, I_{y p y p}=I_{\max }$, together with the respective orientations (Fig.5b).

Taking advantage of the above data, the bending moments about the principal axes were calculated according to Eqs.(1) (Figs.5(c,d)), together with the respective (parasitic) torsional ones, which were calculated according to Eq.(2) (Fig.5d).

$$
\begin{aligned}
& M_{y}=\frac{P_{x} L}{4}, M_{x}=\frac{P_{y} L}{4} \\
& M_{\text {torsional }}=P e
\end{aligned}
$$

Applying now the concepts of the Bernoulli-Euler technical bending theory (and according to the specific conventions adopted for the bending moments in Fig. 5d) the neutral line (zero normal stress line) is determined, according to Eq.(3), as it is demonstrated in Fig.6a for the specific bone (specimen) presented here.

$$
\sigma_{b e n d i n g}=0 \Rightarrow \frac{M_{x}}{I_{x_{p} x_{p}}} y+\frac{M_{y}}{I_{y_{p} y_{p}}} x=0
$$




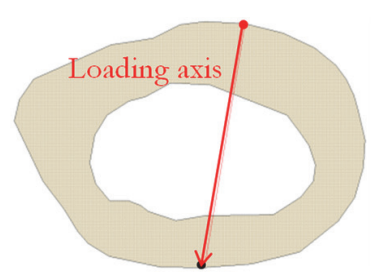

(a)

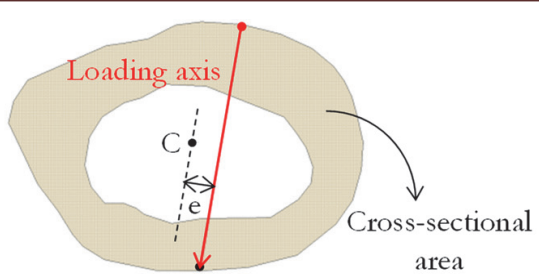

(b)

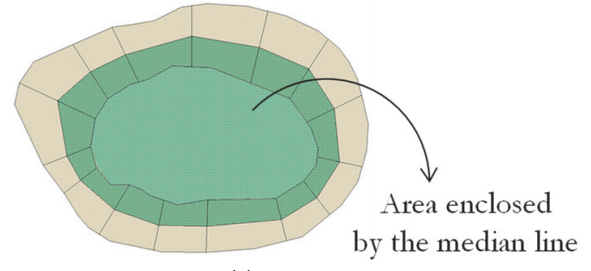

(c)

Figure 4: (a) The "imprint" of the cross-section of a typical bone, as it was obtained using the AutoCAD software; (b) The centroid of the cross-section and the eccentricity of the loading axis; (c) The thickness measured in various locations of the cross-section in order to determine the cortical bone's average thickness and, finally, the area needed for the calculation of the shear stress.

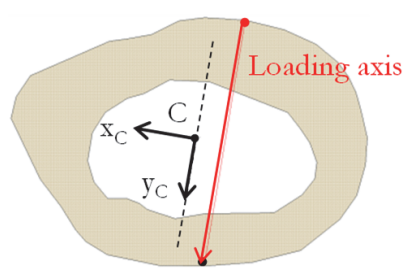

(a)

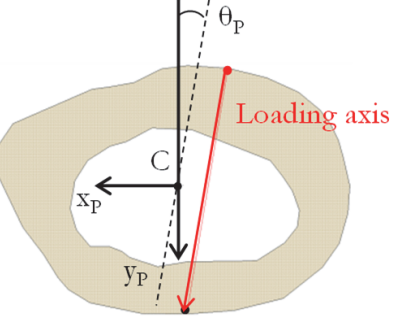

(b)

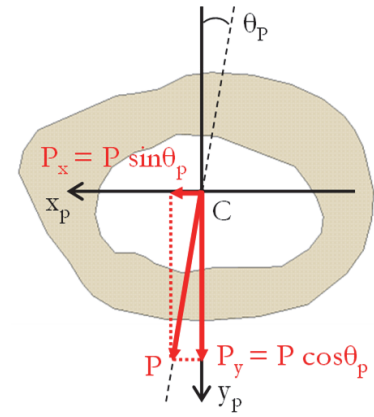

(c)

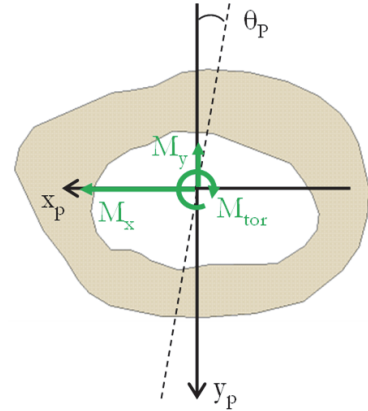

(d)

Figure 5: (a) The auxiliary centroidal system initially chosen arbitrarily (the loading axis is, also, shown); (b) The principal centroidal system determined; (c) The components of the applied load (analyzed along the principal centroidal axes); (d) The bending and the torsional moments developed.

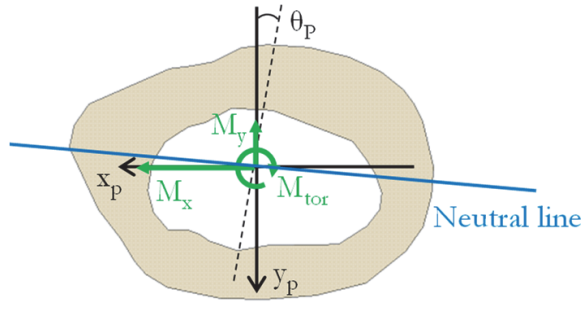

(a)

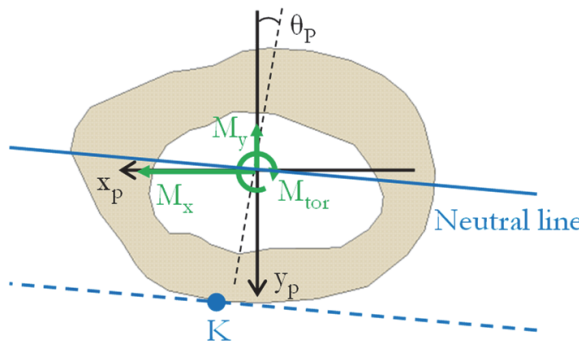

(b)

Figure 6: (a) Determination of the neutral line (zero normal stress locus); (b) Locating the point most distanced from the neutral line, at which the maximum normal (tensile) stress is developed.

Then, as a last step, the maximum normal (tensile) stress at point K, i.e., the most distanced one from the neutral line (Fig. $6 \mathrm{~b})$, is calculated, together with the respective (parasitic) shear stress, according to Eqs. $(4,5)$, where $\mathrm{x}_{\mathrm{k}}$ and $\mathrm{y}_{\mathrm{k}}$ represent the coordinates of point $\mathrm{K}$.

$$
\begin{aligned}
\sigma_{\text {bending }} & =\frac{M_{x}}{I_{x_{p} x_{p}}} y_{k}+\frac{M_{y}}{I_{y_{p} y_{p}}} x_{k} \\
\tau_{\text {torsional }} & =\frac{M_{\text {torsional }}}{2 A_{m} t}
\end{aligned}
$$

It is clarified at this point that the stresses calculated with the aid of Eq.(5) are caused by the torsional moment developed due to the inevitable eccentricity of the loading axis with respect to the centroid of the cross section. On the contrary, the shear stresses due to the internal resultant shear force, $\mathrm{V}$ (stresses provided by the familiar formula $\tau=V Q / \mathrm{It}$, where $\mathrm{I}$ is the respective second moment of area of the cross section, Q is the first moment of area of the part of the cross section located above or below the point at which the stresses are calculated, and the width of the section at the specific point) were ignored, given that the length over "height" ratio exceeds well the limit of 4 (below which these stress are worth being taken into account). 


\section{RESULTS}

$\mathrm{B}$ efore proceeding to the discussion of the results obtained from this study, it is mentioned that the mean values of all quantities (either measured or calculated, tabulated in the respective tables of this section) were obtained after rejecting experiments that were suspicious for biased errors, according to Chauvenet's criterion. In addition, the percentage difference of all quantities between the groups is added in the tables next to the respective mean value. Specifically, the data of the row corresponding to the diabetic rats are compared to the control ones while the data for the diabeticsitagliptin group are compared to both the control and the diabetic animals. For this reason, two percentage differences are presented in the row corresponding to the diabetic-sitagliptin group: the first number in the parenthesis is the comparison to the control group and the second one is the comparison to the diabetic group.

The mean values of the geometrical characteristics of the bones tested, i.e., their cross-sectional area and their average thickness, are presented in Tab. 2 for the three groups of animals tested. The first conclusion that can be drawn is that the cross-sectional area of the diabetic femora is $16 \%$ smaller than the respective one of the control bones. In addition, the bones of the diabetic animals are 13\% thinner than the control ones. On the other hand, both the cross-sectional area and the thickness of the animals treated with sitagliptin are even smaller compared to the respective quantities of the diabetic rats. The average thickness seems to be the quantity most affected.

\begin{tabular}{ccc}
\hline Group & $\begin{array}{c}\text { Cross-sectional area } \\
{\left[\mathrm{mm}^{2}\right]}\end{array}$ & $\begin{array}{c}\text { Average thickness } \\
{[\mathrm{mm}]}\end{array}$ \\
Control & 7.846 & 0.81 \\
Diabetic & $6.581(-16.0 \%)$ & $0.71(-13.0 \%)$ \\
Diabetic-Sitagliptin & $6.466(-18.0 \% /-1.7 \%)$ & $0.66(-18.0 \% /-7.0 \%)$ \\
\hline
\end{tabular}

Table 2: The geometrical characteristics of the groups tested.

\begin{tabular}{ccc}
\hline Group & $\begin{array}{c}\text { Deflection at max load } \\
{[\mathrm{mm}]}\end{array}$ & $\begin{array}{c}\text { Stiffness } \\
{[\mathrm{N} / \mathrm{mm}]}\end{array}$ \\
Control & 0.346 & 576.37 \\
Diabetic & $0.300(-13.0 \%)$ & $670.13(+16.0 \%)$ \\
Diabetic-Sitagliptin & $0.295(-14.5 \% /-1.7 \%)$ & $644.38(+12.0 \% /-3.8 \%)$ \\
\hline
\end{tabular}

Table 3: The deflection at the maximum load and the stiffness of all groups.

Similar conclusions can be drawn concerning the deflection measured at the maximum load attained (Tab. 3). The diabetic femora were definitely proven to be more brittle than the control ones (their deflection was $13 \%$ smaller) and the treatment with sitagliptin seems not to "cure" the bone. On the contrary, the bones belonging to the diabetic-sitagliptin group were slightly more brittle.

It is here recalled, that the deflection of the femora was measured using the video-extensometer (as it was already mentioned previously). The advantage of using this technique is that actual deflection of the femur is provided relieved from any parasitic influences due to deformation of any other element/component of the experimental set-up etc. The astonishing difference between the deflection measured by the video-extensometer and that provided directly by the software of the loading frame is shown in Fig.7a. It is seen that for the specific specimen, the difference between the deflections provided by the extensometer and that provided by the loading frame approaches $40 \%$. It is mentioned, however, that even higher differences have been reported in the literature [10].

Typical load-deflection curves of all three groups tested are shown in Fig.7b. Based on these plots, the stiffness of each femur was calculated. Contrary to what was observed for the three quantities described up to this point (i.e., the crosssectional area, the average thickness and the deflection at the maximum load), diabetes mellitus increases about $16 \%$ the stiffness of the diabetic bones when they are compared to the control ones as it can be seen in Tab. 3. In addition, the use of sitagliptin seems to decrease the stiffness (about $4 \%$ compared to the diabetic femora) approaching to some extent the respective one of the control rats.

Interesting results were obtained concerning the maximum load attained by each group of animals. As it is seen in Tab. 4, the diabetic femora sustain 7\% larger load when they are compared to the maximum load sustained by the femora of the control group. On the other hand, the maximum load recorded for the bones that were treated with sitagliptin is only slightly $(1.5 \%)$ higher compared to that of the control ones. 


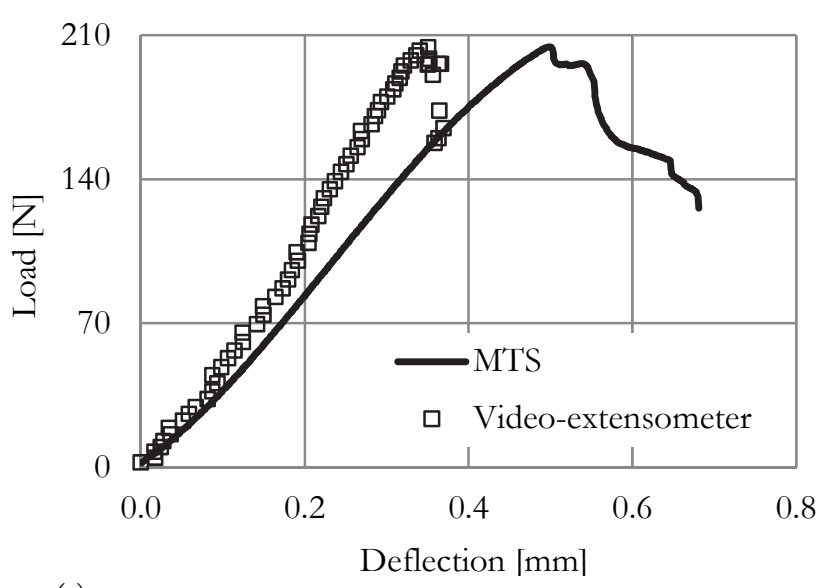

(a)

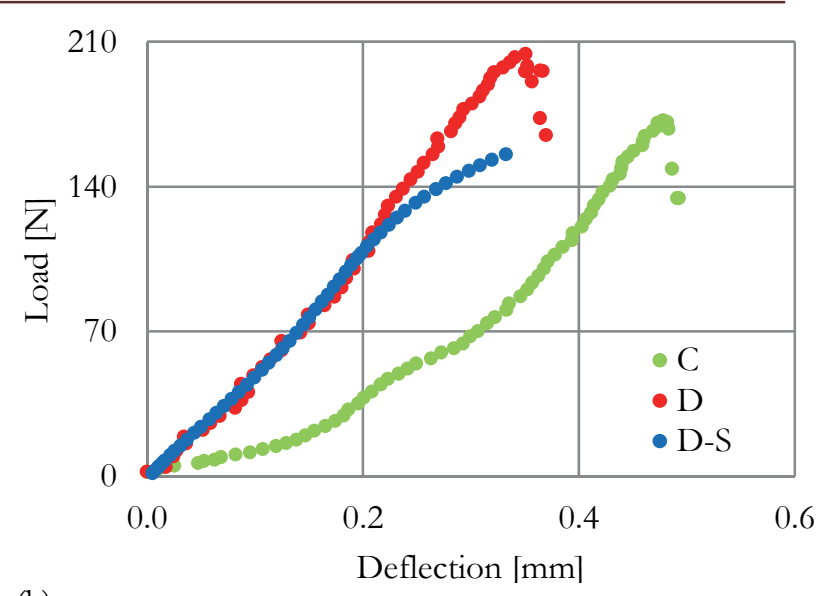

(b)

Figure 7: (a) The load-deflection curves obtained by the video-extensometer and the loading frame for a typical diabetic femur; (b) typical load-deflection curves for the three animal groups of the present study.

\begin{tabular}{ccccc}
\hline Group & Load & Bending strength & Shear stress & Equivalent stress \\
{$[\mathrm{MPa}]$} & {$[\mathrm{MPa}]$} & 211.20 \\
Control & 173.63 & 211.04 & 3.71 & 6.26 \\
Diabetic & $186.44(+7.0 \%)$ & $268.53(+27.0 \%)$ & 7.16 & $268.83(+27.0 \%)$ \\
Diabetic-Sitagliptin & $176.31(+1.5 \% /-5.4 \%)$ & $247.29(+17.0 \% /-7.9 \%)$ & $24.69(+17.0 \% /-7.9 \%)$ \\
\hline
\end{tabular}

Table 4: The load and the stresses developed in the three groups tested.

Similar results, but with greater percentage difference, are observed concerning the bending stress calculated. Surprisingly enough, the bending strength of the diabetic rats seems to be increased with respect to the ones of the control group by about $27 \%$. Moreover, the use of sitagliptin only partly reverses the effect of diabetic mellitus, leading to $17 \%$ greater bending strength compared to the control bones.

The same differences can be seen for the equivalent von Mises stresses. This was expected since the shear stresses produced by the torsional moment developed were proven not significant in this protocol (their magnitude was of the order of about $2.5 \%$ of the respective equivalent stress) and therefore they do not distort the conclusions drawn based on the normal stress. It is herewith recalled that the shear stresses due to the internal resultant shear force were ignored in the analysis of the results on the basis of purely geometrical arguments (the length over "height" ratio of the specimens tested definitely exceeds the critical limit of 4, beyond which the specific "family" of shear stresses are of ignorable magnitude compared to the respective normal stresses).

\section{DisCussion}

he target of the present study was two-folded: On the one hand it was attempted to quantify the potentially harmful role of Type 2 Diabetes Mellitus on the biomechanical properties of the bone tissue. On the other hand, it was attempted to check whether some of these damages can be restored with the aid of a widely used treatment against diabetes, based on sitagliptin. To achieve these targets, three groups of male 10-week old Wistar rats were used (control, diabetic and diabetic treated with sitagliptin).

Taking into account that some of the findings of the experimental protocol (especially the ones concerning the relation between geometric characteristics and mechanical properties) could be considered as "unexpected", an attempt to gain deeper insight into the role of T2DM and sitagliptin is undertaken. In this direction two critical geometrical characteristics of the femora, i.e., the cross-sectional area and the second principal moments of area, were considered against the respective average value of the weight of the animals of the three groups.

The results of this correlation are plotted in Figs.8a for the cross sectional area and in Fig.8b for the second moment of area. It is definitely concluded that T2DM significantly contributes to loss of weight of the sufferers. Treating them with sitagliptin increases further the loss of weight as it is also mentioned by other researchers [16, 17]. Similarly, T2DM decreases the second moments of area. Sitagliptin does not reverse this trend. 


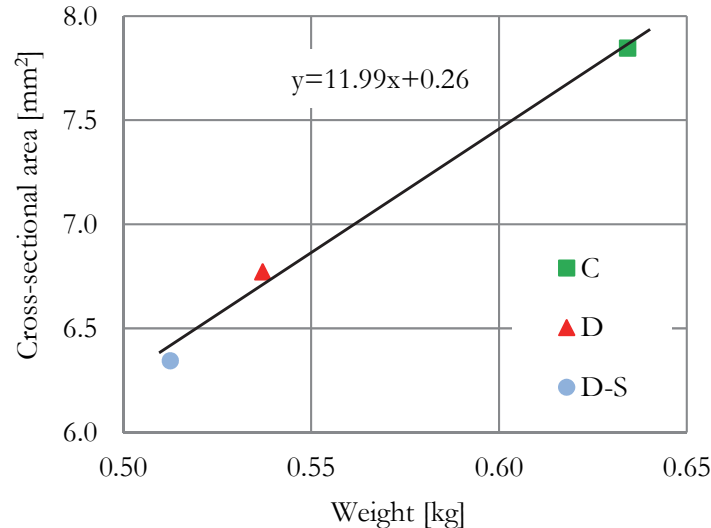

(a)

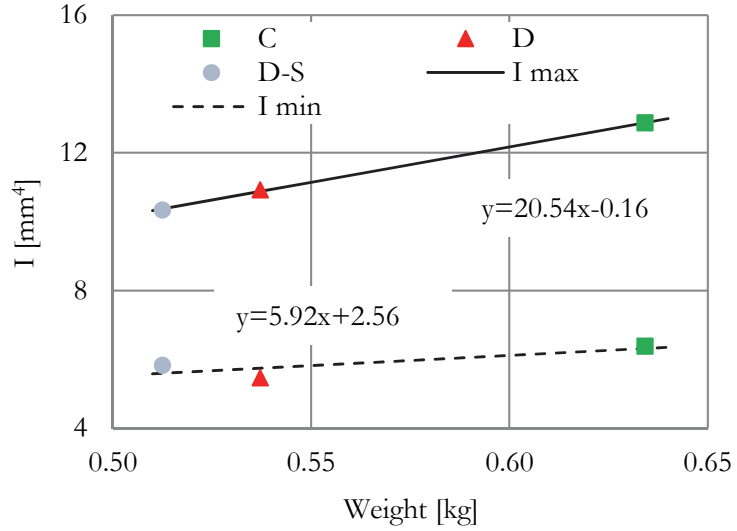

(b)

Figure 8: The average weight of the animals of the three groups versus the respective average value of (a) The femora's cross-sectional area and (b) The femora's principal second moments of area.

At this point it is obvious that a kind of paradox comes into fora: While the geometrical characteristics of diabetic bone tissue are considerably downgraded, the respective load carrying capacity increases. Obviously this controversial behaviour cannot be explained on purely engineering terms. Histopathological studies, in parallel with the respective biomechanical ones, seems to be "sine qua non" in order to clearly understand the damage mechanisms activated by T2DM.

\section{CONCLUDING REMARKS}

he basic conclusions drawn from this study can be categorized into two groups, i.e., one concerning the geometrical features of the critical cross section of the femora and a second one concerning the mechanical properties of the specific bone as a structure under mechanical load. Concerning geometry, it was concluded that the cross-sectional area of diabetic femora is almost $16 \%$ smaller compared to that of the control bones. Moreover, the bone of diabetic animals is about 13\% thinner than that of the control ones. For the diabetic animals treated with sitagliptin the cross-sectional area and the thickness of the bone are even smaller compared to the respective quantities of diabetic rats.

Concerning the mechanical properties and parameters, it is to be accepted that some of the conclusions drawn from the present protocol are not in full accordance with what was perhaps expected. Namely, the fact that the femora of diabetic animals sustain $7 \%$ higher load with respect to that of the control group, was, indeed, somehow unexpected. One could anticipate that this increase could be just the result of increased cross-sectional area (or increased second moment of area) of the femora of diabetic rats, but this is not the case. Expressing the fracture load in terms of the respective normalized quantity (ultimate normal bending stress or bending strength) provides even more surprising results: The bending strength of the femora of the diabetic animals appears almost $27 \%$ higher than that of the femora of the animals of the control group. Along the same lines, it seems that diabetes mellitus increases the stiffness (about 16\%) of the bone tissue with respect to that of the control animals. Again, sitagliptin does not bring things in their initial status (i.e., to that of the control group): Although the bone tissue stiffness of animals treated with sitagliptin is almost $4 \%$ lower compared to that of the diabetic animals it is still almost $12 \%$ higher compared to that of the control animals.

The above conclusions may sound peculiar but this is not the first time that similar findings are reported in international literature. For example Prisby et al. [11], as well as Reinwald et al. [12], observed a similar behaviour. On the contrary, other researchers have reported opposite experimental results indicating either negative role of T2DM on bone quality [13, 14] or negligible influence $[12,15]$.

In any case, it is beyond discussion that the role of T2DM on the quality of the bone tissue must be further studied before definite conclusions are drawn. The current consensus in the medical community is that patients with T2DM have 40-70\% increased fracture risk (which in terms of Mechanics of Materials could be translated to increased brittleness); however, their bone mineral density is normal or increased (which, again in terms of Mechanics of Materials could be translated to increased bending strength). This suggests that there are other factors besides bone quality that affect bone fragility. It is here mentioned that this is not the case for patients with type I diabetes, who are characterized by low bone mineral density and a six-fold increased fracture risk, implying that chronic hyperglycemia is not the main pathophysiological mechanism 
of increased fracture risk in diabetic patients $[4,5]$. The above consensus concerning brittleness was definitely supported by the present protocol. Indeed, it was indicated that the deflection at the maximum load of the diabetic femora was $13 \%$ smaller compared to that of the control group. Unfortunately, it was concluded (also beyond any doubt) that sitagliptin does not cure the problem of increased brittleness of diabetic bone tissue.

\section{REFERENCES}

[1] Nauck, M.A. and Meier, J.J. (2018). Incretin hormones: Their role in health and disease, Diabetes Obes. Metab., 20, pp. 5-21. DOI: 10.1111/dom.13129.

[2] Janghorbani, M., Van Dam, R.M., Willett, W.C. and Hu, F.B. (2007). Systematic review of type 1 and type 2 diabetes mellitus and risk of fracture. Am. J. Epidemiol., 166, pp. 495-505. DOI: 10.1093/aje/kwm106.

[3] Napoli, N., Chandran, M., Pierroz, D.D., Abrahamsen, B., Schwartz, A.V., Ferrari, S.L. and IOF Bone and Diabetes Working Group (2017). Mechanisms of diabetes mellitus-induced bone fragility, Nat. Rev. Endocrinol., 13, pp. 208219. DOI: $10.1038 /$ nrendo.2016.153.

[4] Hofbauer, L.C., Brueck, C.C., Singh, S.K. and Dobnig, H. (2007). Osteoporosis in patients with diabetes mellitus, J. Bone Miner. Res., 22, pp. 1317-1328. DOI: 10.1359/jbmr.070510.

[5] Napoli, N., Strotmeyer, E.S., Ensrud, K.E. et al. (2014). Fracture risk in diabetic elderly men: the MrOS study, Diabetologia, 57, pp. 2057-2065. DOI: 10.1007/s00125-014-3289-6.

[6] Purnamasari, D., Puspitasari, M.D., Setiyohadi, B., Nugroho, P. and Isbagio, H. (2017). Low bone turnover in premenopausal women with type 2 diabetes mellitus as an early process of diabetes-associated bone alterations: a crosssectional study, BMC Endocr. Disord., 17, Article number: 72. DOI: 10.1186/s12902-017-0224-0.

[7] Krakauer, J.C., McKenna, M.J., Buderer, N.F., Rao, D.S., Whitehouse, F.W. and Parfitt, A.M. (1995). Bone loss and bone turnover in diabetes, Diabetes, 44, pp. 775-782. DOI: 10.2337/diab.44.7.775.

[8] Biewener, A.A. (1982). Bone strength in small mammals and bipedal birds: Do safety factors change with body size?, J. Exp. Biol., 98, pp. 289-301.

[9] Yabe, D. and Seino, Y. (2013). Incretin actions beyond the pancreas: Lessons from knockout mice, Curr. Opin. Pharmacol., 13, pp. 946-953. DOI: 10.1016/j.coph.2013.09.013.

[10] Kourkoulis, S.K., Kouvaka, A., Andriakopoulou, Ch. and Dontas, I. (2016). An alternative approach for the interpretation of data from three-point bending of long bones, Engineering Transactions, 64(4), pp. 401-407.

[11] Prisby, R.D., Swift, J.M., Bloomfi eld, S.A., Hogan, H.A. and Delp, M.D. (2008). Altered bone mass, geometry and mechanical properties during the development and progression of type 2 diabetes in the Zucker diabetic fatty rat, $\mathrm{J}$ Endocrinol., 199(3), pp. 379-388. DOI: 10.1677/JOE-08-0046.

[12] Reinwald, S., Peterson, R.G., Allen, M.R. and Burr, D.B. (2009). Skeletal changes associated with the onset of type 2 diabetes in the ZDF and ZDSD rodent models, Am. J. Physiol. Endocrinol. Metab., 296(4), pp. E765-774. DOI: 10. 1152/ajpendo.90937.2008.

[13] Gallant, M.A., Brown, D.M., Organ, J.M. et al. (2013). Reference-point indentation correlates with bone toughness assessed using whole-bone traditional mechanical testing, Bone, 53(1), pp. 301-305. DOI: 10.1016/j.bone.2012.12.015.

[14] Hamann, C., Rauner, M., Höhna, Y. et al. (2013). Sclerostin antibody treatment improves bone mass, bone strength, and bone defect regeneration in rats with type 2 diabetes mellitus, J. Bone Miner. Res., 28(3), pp. 627-638. DOI: 10. 1002/jbmr.1803.

[15] Hill Gallant, K.M., Gallant, M.A., Brown, D.M. et al. (2014). Raloxifene prevents skeletal fragility in adult female Zucker Diabetic Sprague-Dawley rats, PLoS One, 9(9), e108262. DOI: 10.1371/journal.pone.0108262.

[16] Gül, Ö.Ö., Kıyıc1, S., Ersoy, C. et al. (2011). Effect of sitagliptin monotherapy on serum total ghrelin levels in people with type 2 diabetes, Diabetes Res. Clin. Pr., 94(2), pp. 212-216. DOI: 10.1016/j.diabres.2011.07.031.

[17] Yanai, H., Adachi, H., Hamasaki, H. et al. (2012). Effects of 6-month sitagliptin treatment on glucose and lipid metabolism, blood pressure, body weight and renal function in type 2 diabetic patients: a chart-based analysis, J. Clin. Med. Res., 4(4), pp. 251-258. DOI: 10.4021/jocmr975w. 